Pacific Journal of Mathematics

HOMOMORPHISMS AND SUBDIRECT DECOMPOSITIONS OF 


\title{
HOMOMORPHISMS AND SUBDIRECT DECOMPOSITIONS OF SEMIGROUPS
}

\author{
B. M. SchEIN [Š́̆ \\ Dedicated to Professor Alexander Doniphan Wallace \\ on the occasion of his sixtieth birthday
}

Subdirect decompositions of rings seem to be an important tool in the theory of rings promoting the development of this theory. It is a very natural thing to study subdirect products of semigroups but to the author's knowledge the only paper on the topic is that of $G$. Thierrin [22] where certain properties of subdirectly irreducible semigroups are considered.

Subdirect decompositions of semigroups are closely connected with homomorphisms of these semigroups, so we describe in the first section the structure of an arbitrary congruence on a semigroup. The second section is devoted to certain special subsets and elements of a semigroup. Main notions of the section are those of disjunctive element (i.e., an element that does not form a congruence class modulo any nontrivial congruence) and of core of a semigroup (i.e., a least nonnull ideal). Subdirectly irreducible semigroups are considered in the third, fourth and fifth sections. We consider certain general properties of such semigroups and find characterizations of special classes of such semigroups (e.g. nilpotent, idempotent, commutative). Section 6 treats homomorphically simple ( $h$-simple) semigroups, i.e., semigroups having no nontrivial congruences. Section 7 is devoted to consideration of certain semigroups having special subdirect decompositions. By analogy with $f$-regular rings [3] we introduce $f$-regular semigroups. There are considered also completely reductive semigroups, i.e., semigroups having no nononreductive homomorphic images.

Several results of this paper have been published without proofs in our note [18]. Certain results of [18] had been previously found in [22] but we did not know this when [18] was published. All concepts of the theory of semigroups that are not defined here are defined in $[6,12]$. We use the symbols $\Lambda, \rightarrow, \leftrightarrow, \Lambda$ respectively for conjunction, implication, (logical) equivalence, universal quantifier and follow the ordinary agreement as to the use of brackets in statements. If $\varepsilon$ is an equivalence relation, then $\varepsilon\langle g\rangle$ is the $\varepsilon$-class containing $g$ and $g_{1} \equiv g_{2}(\varepsilon)$ or $g_{1} \equiv g_{2}$ means that $g_{1}$ and $g_{2}$ are in the relation $\varepsilon$. If $G$ is a semigroup then $G^{1}$ denotes $G$ with adjoined identity (unless $G$ already has an identity), $G^{\circ}$ denotes $G$ with adjoined zero (unless $G$ already has a zero). Variables $g$ and $h$ (with or without indices) take values in the set of all elements 
of $G$, variables $x$ and $y$ take values in the set of all elements of $G^{1}$ (i.e., $x$ and $y$ may be void symbols ([12], p. 7)). A oneelement set is often denoted in the same way as its element. As a rule, one-element semi-groups are excluded from consideration. $\Delta_{G}$ is the identity relation on the set $G$.

Congruences on semigroups. It is known that the consideration of homomorphisms may be limited to the consideration of congruences on semigroups (it is necessary to emphasize that such a limitation can lead to an essential loss of information-e.g., when one considers automorphisms).

Let $\theta(G)$ denote the complete lattice of all congruences on a semigroup $G$. A minorant basis of $\Theta(G)$ is any subset $B$ of $\Theta(G)$ such that each element of $\theta(G)$ is the greatest lower bound (i.e., the intersection) of some subset of $B$.

If $H$ is a subset of a semigroup $G$ then the equivalence $\mathscr{C}_{H}$ defined as follows:

$$
g_{1} \equiv g_{2}\left(\mathscr{C}_{H}\right) \leftrightarrow(\Lambda x, y)\left[x g_{1} y \in H \leftrightarrow x g_{2} y \in H\right]
$$

is a congruence $[17,21]$ - the principal congruence determined by H. R. Pierce [14] and R. Croisot [7] define $\mathscr{C}_{H}$ in another way (they do not allow $x$ and $y$ in (1.1) to be void). Clearly $\mathscr{C}_{H}=\mathscr{C}_{H^{\prime}}$, where $H^{\prime}$ is the complementation of $H$ in G. E.g., $\mathscr{C}_{\phi}=\mathscr{C}_{G}=G \times G$.

Let $\varepsilon$ be an equivalence on a semigroup $G$. The greatest congruence included in $\varepsilon$ is called the stable opening of $\varepsilon$ (cf. [16]). We omit the straightforward proof of the following

Lemma 1.1. Every equivalence $\varepsilon$ on a semigroup $G$ possesses a stable opening $\bar{\varepsilon}$ and $\bar{\varepsilon}=\bigcap\left(\mathscr{C}_{\varepsilon\langle g\rangle}\right)_{g \in G}$.

Every congruence coincides with its stable opening, so every congruence $\varepsilon$ coincides with intersection of the family of principal congruences generated by $\varepsilon$-classes.

THEOREM 1.2. The set of all principal congruences on a semigroup forms a minorant basis of the lattice of all congruences.

This theorem is no longer true if principal congruences are understood in the sense of $R$. Croisot [7].

Let $G$ be a subsemigroup of a ring $R$. Then every congruence of the ring $R$ induces a congruence on $G$. It is easy to prove that if $R$ is the semigroup ring of $G$ over the ring of integers, then every congruence of $G$ is induced by some congruence of $R$. 
2. Disjunctive element and cores of semigroups. A subset $H$ of a semigroup $G$ is called indivisible by an equivalence $\varepsilon$ (by a subset $F$ ) if $H$ is contained in some $\varepsilon$-class ( $\mathscr{C}_{F}$-class). $H$ is called saturated for $\varepsilon$, if $H$ is the union of a family of $\varepsilon$-classes. M. Teissier [21] has proved that $\mathscr{C}_{H}$ is the greatest congruence for which $H$ is saturated.

Let us associate with every subset $H$ the subset $r(H)$ defined by the formula

$$
g \in \boldsymbol{r}(H) \leftrightarrow(\bigwedge x, y)[x g y \notin H]
$$

$\boldsymbol{r}(H)$ is called the (bilateral) residue of $H$ in $G$. It is an ideal of $G$ and, if nonempty, an $\mathscr{C}_{H}$-class. If $x$ and $y$ in (2.1) are not permitted to be void, one comes to the definition of residue in the sense of R. Croisot [7]. In our previous papers we designated $\mathscr{C}_{H}$ by $\varepsilon$ and

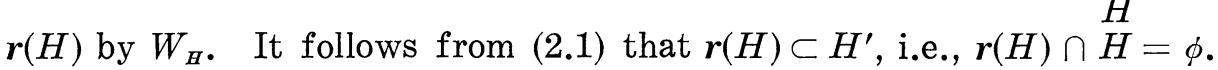
$H$ is called neat if $r(H)=\phi$. If $G$ contains a zero 0 and $0 \notin H$ then $H$ cannot be neat because $0 \in r(H) . \quad H$ is called 0 -neat if $\boldsymbol{r}(H)$ contains at most one element (i.e., $\boldsymbol{r}(H)$ coincides with $\phi$ or with $\{0\}$ ). Both notions are identical for a semigroup without zero.

A subset $H$ is called disjunctive if the only subsets indivisible by $\mathscr{C}_{H}$ are empty and one-element. This means that

$$
\mathscr{C}_{H}=\Delta t
$$

Disjunctive subsets were considered by E. J. Tully, Jr. [23], M. P. Schützenberger [20] and (in a slightly different sense) by R. Pierce [14].

An element $g$ is called 0-neat (disjunctive) if $\{g\}$ is 0 -neat (disjunctive). In [18] disjunctive elements were called separative.

The least nonempty ideal of a semigroup $G$ (if it exists) is called the kernel of $G$. The kernel of a semigroup with zero is trivial. We call an ideal nonnull if it contains at least two elements. The least nonnull ideal (if it exists) is called the core of $G$. The core and the kernel of a semigroup without zero coincide.

THEOREM 2.1. Let $G$ be a semigroup with disjunctive zero. Then an element of $G$ is disjunctive if and only if it is 0-neat.

Proof. If an element $k$ is disjunctive then by (2.2) $\boldsymbol{r}(k)$ contains at most one element. Hence $k$ is 0 -neat. Now let $k$ be 0 -neat. If $k=0$ then $k$ is disjunctive, so let $k \neq 0$. For every $g_{1}, g_{2} \in G$ there exist $x, y$ such that exactly one of elements $x g_{1} y, x g_{2} y$ is equal to 0 (this follows from disjunctivity of 0 ). Let $x g_{1} y \neq 0$. Then there 
exist $x_{1}, y_{1}$ such that $x_{1} x g_{1} y y_{1}=k$. But $x g_{2} y=0$, so $x_{1} x g_{2} y y_{1}=0 \neq k$, and $g_{1} \not \equiv g_{2}\left(\mathscr{C}_{k}\right)$, i.e., $k$ is disjunctive.

Let $K$ be the core of $G$. Since $K^{2}$ is an ideal, one has two alternatives: $K^{2}=K$ or $K^{2}=0$. In the first case we call $K$ globally idempotent, in the second case $K$ is called nilpotent.

The set of all right annihilating elements of a semigroup $G$ (i.e., such elements $g$ that $G g=0$ ) is called the right annihilator of $G$. The left annihilator is defined dually. The set of all (left and right) annihilating elements is called the annihilator of $G$. The right annihilator is called trivial if it contains at most one element.

A core $K$ is called primitive if it contains exactly one nonzero element.

THEOREM 2.2. If a semigroup $G$ having a core $K$ contains a nonzero element $g$ such that $G g G=0$, then $K$ is included in the right or in the left annihilator of $G$. If $K$ is included in both, then $K$ is primitive and coincides with the annihilator. In every case the core $K$ is nilpotent.

Proof. Let $M(G)$ be the set of all $g \in G$ such that $G g G=0$. $M(G)$ is a nonnull ideal, so $K \subset M(G)$ and $G K G=0$. Therefore $G K$ and $K G$ are ideals. If $G K$ is nonnull, then $K \subset G K$, so $G K=K$ and $K G=0$, i.e., $K$ is contained in the left annihilator. Analogously, if $K G$ is nonnull, then $K$ is contained in the right annihilator. If $G K=$ $K G=0$ then $K$ is contained in the annihilator. Every subset of the annihilator is an ideal, hence the annihilator cannot contain more than two elements. So $K$ coincides with the annihilator and is primitive. Clearly, in every case $K^{2}=0$.

It is easy to prove that if the core $K$ is globally idempotent then $K g K=K$ for every nonzero $g \in G$.

3. General properties of subdirectly irreducible semigroups. Let $\left(G_{i}\right)_{i \in I}$ be a nonempty family of semigroups. Its direct product is a semigroup $\times\left(G_{i}\right)_{i \in I}$ whose set of elements is the Cartesian product of the family of sets of elements $G_{i}$, the operation is defined componentwise.

A subsemigroup $G$ of the semigroup $\times\left(G_{i}\right)$ is called a subdirect product of the family $\left(G_{i}\right)_{i \in I}$ of semigroups if $\operatorname{pr}_{i}(G)=G_{i}$ for all $i \in I$ (here $p r_{i}$ denotes the natural projection of $\times\left(G_{i}\right)$ on $G_{i}$ ). Clearly, all $p r_{i}$ are homomorphic mappings of $G$.

We say that a semigroup $S$ is decomposable as a subdirect product of a family $\left(G_{i}\right)_{i \in I}$ of semigroups if $S$ is isomorphic to the subdirect 
product of $\left(G_{i}\right)_{i}$. This isomorphism is called a subdirect decomposition of $S$.

If the subdirect decomposition followed by some one of the projections $p r_{i}$ is an isomorphism of $S$ with $G_{i}$, then this decomposition is called trivial. A semigroup is called subdirectly irreducible if each of its subdirect decompositions is trivial. In the same way direct decompositions and directly irreducible semigroups are defined.

Two following theorems are true ([2], Th. 10, p. 92; [1], p. 765):

THEOREM 3.1. Every semigroup is decomposable as a subdirect product of a family of subdirectly irreducible semigroups.

THEOREM 3.2. A semigroup $G$ is subdirectly irreducible if and only if it possesses the least nonidentical congruence.

If a family $\left(\varepsilon_{i}\right)$ of congruences on $G$ has $\Delta_{G}$ as its intersection, then $G$ is decomposable as a subdirect product of a family $\left(G / \varepsilon_{i}\right)$ of semigroups ([2], Th. 9, p. 92).

THEOREM 3.3. Every subdirectly irreducible semigroup has at least two different disjunctive elements.

Proof. Consider the congruence $\cap\left(\mathscr{C}_{g}\right)_{g \in \theta}$ on a subdirectly irreducible semigroup $G$. Each of $\{g\}$ is a $\mathscr{C}_{g}$-class, so our congruence is equal to $\Delta_{G}$. Since $G$ is subdirectly irreducible, $\mathscr{C}_{g_{1}}=\Delta_{G}$ for some $g_{1}$ (by Theorem 3.2). Now consider the congruence $\bigcap\left(\mathscr{C}_{g}\right)_{g \neq g_{1}}$. Every $g \neq g_{1}$ forms its congruence class, so our congruence is equal to $\Delta_{G}$ and there exists $g_{2} \neq g_{1}$ such that $\mathscr{C}_{g_{2}}=\Delta_{G} \cdot \quad g_{1}$ and $g_{2}$ are two different disjunctive elements, by (2.2).

Disjunctive elements are 0-neat, every nonzero 0-neat element belongs to every nonnull ideal, i.e., belongs to a core.

COROLLARY 3.3.1. [22]. Every subdirectly irreducible semigroup has a core.

All disjunctive elements of a semigroup belong to its core. But a semigroup having a core need not be subdirectly irreducible (as it is for rings).

THeOREM 3.4. Let $\mathscr{H}$ be a family of subsets of a subdirectly irreducible semigroup and let the intersection $\cap H$ or the union $\mathrm{U} H$ of this family be disjunctive. Then at least one subset in the family $\mathscr{H}$ is disjunctive. 
Proof. It is easy to prove (cf. [17], p. 387) that

$$
\bigcap\left(\mathscr{C}_{H_{i}}\right)_{i \in I} \subset \mathscr{C}_{n\left(H_{i}\right)_{i} \in I}, \quad \bigcap\left(\mathscr{C}_{H_{i}}\right)_{i \in I} \subset \mathscr{C}_{\mathbf{U}\left(H_{i}\right)_{i} \in I} .
$$

If $\mathscr{H}=\left(H_{i}\right)$ and $\bigcap \mathscr{H}$ or $\bigcup H$ are disjunctive, then $\bigcap\left(\mathscr{C}_{H_{i}}\right)=\Delta_{G}$. Hence there exists $i \in I$ such that $\mathscr{C}_{H_{i}}=\Delta_{G}$, i.e., $H_{i}$ is disjunctive.

COROLlARY 3.4.1. Let $G$ be a subdirectly irreducible semigroup, $K$ its core, $\varepsilon_{0}$ the least nonidentical congruence on $G, k$ a disjunctive element of $G, H \subset G$. Every disjunctive subset of $G$ contains at least one disjunctive element. $H$ is saturated for $\varepsilon_{0}$ if and only if it is not disjunctive. $H$ is disjunctive if and only if $H \cap K$ is such. If $g \in G$ and $\{k, g\}$ is not disjunctive, then every subset of $G$ containing $k$ but not $g$ is disjunctive; excluding at most one, every two-element subset containing $k$ is disjunctive.

This follows from Theorem 3.4 and evident equality $H=$ $(H \cap K) \cup(H \backslash K)$. If $\{k, g\}$ is not disjunctive then every subset containing $k$ but not $g$ intersects with $\{k, g\}$ by $\{k\}$.

Let $g, h$ be elements of a semigroup $G$. If $g h=h$ then $g$ is called a left unit for $h$. If $g h=h g=h$, then $g$ is called a unit for $h$. An element $e$ is called central if $e g=g e$ for every $g \in G$.

THEOREM 3.5. Let $G$ be a subdirectly irreducible semigroup and $e$ a central element of $G$. If $e$ is a unit for some nonzero element then $e$ is the identity of $G$.

Proof. Let $e$ be a unit for $g \neq 0, K$ be the core of $G$ and $k \in K$. There exist $x, y$ such that $x g y=k$ (since $k$ is 0 -neat), so $k e=k$, since $e$ is central. So for every $k \in K k e=k$ and for an arbitrary positive $n k e^{n}=k$. Consider a binary relation $\varepsilon_{(e)}$ defined by the formula: $g_{1} \equiv g_{2}\left(\varepsilon_{(e)}\right) \leftrightarrow g_{1} e^{m}=g_{2} e^{n}$ for some positive integers $m$ and $n$. Clearly, $\varepsilon_{(e)}$ is a congruence and it induces on $K$ the identical congruence. Let $\varepsilon^{K}=K \times K \cup \Delta_{G}$ be the congruence generated by $K$. Then $\varepsilon^{K} \cap \varepsilon_{(e)}=$ $\Delta_{G}$. Since $G$ is subdirectly irreducible and $\varepsilon^{K} \neq \Delta_{G}, \quad \varepsilon_{(e)}=\Delta_{G}$. For every $g \in G g \equiv g e\left(\varepsilon_{(e)}\right)$, so $g=g e$.

Every central idempotent is a unit for itself. So we have:

CoROLLARY 3.5.1. [22]. A subdirectly irreducible semigroup does not contain central idempotents different from zero and identity.

THEOREM 3.6. Semigroups $G$ and $G^{1}\left(G\right.$ and $\left.G^{0}\right)$ are simultaneously subdirectly irreducible or reducible. 
Proof. If $G$ has an identity, then $G=G^{1}$ and there is nothing to prove. Let $G$ be a semigroup without identity. If $\varepsilon$ is a congruence on $G$, then $\varepsilon^{1}=\varepsilon \cup\{(1,1)\}$ is a congruence on $G^{1}$. If $\varepsilon_{0}$ is the least nonidentical congruence on $G^{1}$, then it induces the least nonidentical congruence on $G$. It is easy to prove that if $\varepsilon_{0}$ is the least nonidentical congruence on $G$ then $\varepsilon_{0}^{1}$ is the least nonidentical congruence on $G^{1}$. The proof for $G$ and $G^{0}$ is analogous.

THEOREM 3.7. A semigroup $G$ with disjunctive zero is subdirectly irreducible if and only if it satisfies one of the following conditions (which are equivalent):

(1) $G$ contains at least two different 0-neat elements.

(2) $G$ contains at least two different disjunctive elements.

(3) G has a core.

Proof. By Theorem 2.1, conditions (1)-(3) are equivalent. They are necessary for subdirect irreducibility (Theorem 3.3). Now let $G$ satisfy (1)-(3), $k$ be a 0 -neat element different from 0 . If $\varepsilon$ is a nonidentical congruence, then $\{0\}$ cannot be an $\varepsilon$-class (otherwise $\varepsilon \subset \mathscr{C}_{0}=$ $\left.\Delta_{G}\right)$. So $0 \equiv g(\varepsilon)$ for some nonzero $g$. Since $k$ is 0 -neat there exist $x$ and $y$ such that $x g y=k$, so $0=x 0 y \equiv x g y=k$. This is true for every $k \in K$, so $\varepsilon^{K} \subset \varepsilon$ and the congruence $\varepsilon^{K}$ generated by $K$ is the least nonidentical congruence on $G$. $G$ is subdirectly irreducible, by Theorem 3.2.

CoROLlary 3.7.1. A semigroup with primitive core is subdirectly irreducible if and only if its zero is disjunctive.

A subset $H$ of a semigroup $G$ is called a left reductor if it has the property:

If $g_{1}, g_{2} \in G$ and $h g_{1}=h g_{2}$ for every $h \in H$, then $g_{1}=g_{2}$.

$H$ is a right reductor if it satisfies the dual property.

We define the congruences $\varepsilon_{r}$ and $\varepsilon_{1}$ by the formulas

$$
\begin{aligned}
& g_{1} \equiv g_{2}\left(\varepsilon_{r}\right) \leftrightarrow(\bigwedge g)\left[g_{1} g=g_{2} g\right] \\
& g_{1} \equiv g_{2}\left(\varepsilon_{1}\right) \leftrightarrow(\Lambda g)\left[g g_{1}=g g_{2}\right] .
\end{aligned}
$$

$G$ is called right (left) reductive if $\varepsilon_{r}=\Delta_{G}\left(\varepsilon_{1}=\Delta_{G}\right)$.

Let $G$ be a subdirect product of a family $\left(G_{i}\right)$ of right reductive semigroups and $g_{1} g=g_{2} g$ for every $g \in G$. Then $\operatorname{pr}_{i}\left(g_{1}\right) p r_{i}(g)=$ $p r_{i}\left(g_{2}\right) p r_{i}(g)$. The elements $p r_{i}(g)$ run over the whole set $G_{i}$, so $p r_{i}\left(g_{1}\right)=p r_{i}\left(g_{2}\right)$, i.e., $g_{1}=g_{2}$. We have proved:

THEOREM 3.8. A subdirect product of a family of right (left) reductive semigroups is right (left) reductive. 
Let $G$ be subdirectly irreducible, $K$ its core. Denote the congruence $\varepsilon_{r} \cap \varepsilon_{1} \cap \varepsilon^{K}$ by $\varepsilon$. Let $g_{1} \equiv g_{2}(\varepsilon)$. If $g_{1}$ or $g_{2}$ does not belong to $K$, then $g_{1}=g_{2}$. Let $g_{1}, g_{2} \in K$. Then

$$
(\Lambda g)\left[g_{1} g=g_{2} g \wedge g g_{1}=g g_{2}\right] \text {. }
$$

Let $k$ be disjunctive and different from $g_{1}$ and $g_{2}$. If $x g_{1} y=k$, then $x$ or $y$ is not void, so $x g_{1} y=x g_{2} y=k$. Therefore $g_{1} \equiv g_{2}\left(\mathscr{C}_{k}\right)$, i.e., $g_{1}=g_{2}$. Two alternatives are possible: 1) $\varepsilon=\Delta_{G}$. Therefore $\varepsilon_{r}$ or $\varepsilon_{1}$ is identical, i.e., $G$ is right or left reductive. 2) $\varepsilon \neq \Delta_{G}$. Then there exist $g_{1} \neq g_{2}$ such that $g_{1} \equiv g_{2}(\varepsilon)$. $G$ has no disjunctive elements different from $g_{1}, g_{2}$. So $g_{1}$ and $g_{2}$ are disjunctive (by Theorem 3.3). We have proved:

THEOREM 3.9. If a subdirectly irreducible semigroup is neither right nor left reductive, then it contains exactly two disjunctive elements $g_{1}$ and $g_{2}$ and these elements satisfy (3.4).

4. Special classes of subdirectly irreducible semigroups. A homogroup is a semigroup which contains a kernel that is a group $[5,6]$. A semigroup is a homogroup if and only if the intersection of all right, left and two-sided ideals of the semigroup is not empty. Every semigroup with zero is a homogroup. If $K$ is the kernel of a homogroup $G$ then the identity of the group $K$ is a central idempotent of $G([12]$, p. 252). If $G$ is subdirectly irreducible, then this central idempotent is a zero or an identity of $G$, by Corollary 3.5.1. In the second case $G=K$. So we have:

THEOREM 4.1. Every subdirectly irreducible homogroup without zero is a group.

It follows that a subdirectly irreducible semigroup which is not a group does not contain nonzero zeroid elements in the sense of [5]. It follows also that the core of this semigroup is not a group.

A semigroup is called a nilsemigroup if some power of every element is equal to zero (the power may be different for different elements).

Lemma 4.2. If a nilsemigroup has a core, this core is primitive and coincides with the annihilator of the semigroup.

Proof. Let $G$ be a nilsemigroup and $K$ its core, $k_{1}, k_{2}$ be two nonzero elements of $K$. Since $k_{1}$ and $k_{2}$ are 0 -neat, there exist $x, y$, $x_{1}, y_{1}$ such that $x k_{1} y=k_{2}$ and $x_{1} k_{2} y_{1}=k_{1}$. So $\left(x_{1} x\right)^{n} k_{1}\left(y y_{1}\right)^{n}=k_{1}$ for 
every $n$, i.e., $\left(x_{1} x\right)^{n} \neq 0$ and $\left(y y_{1}\right)^{n} \neq 0$. Hence, $x, y, x_{1}, y_{1}$ are void and $k_{1}=k_{2}$, i.e., $K$ contains a single nonzero element, say, $k$. If $G K=G K \cup\{0\}$ is not equal to 0 , then $G K=K$ and $g k=k$ for some $g \in G$. So $g^{n} k=k$ for every $n$. But $g^{n}=0$ for some $n$. So $G K=0$. Analogously, $K G=0$. Hence $K$ is the annihilator, by Theorem 2.2.

By this lemma and Corollary 3.7.1,

THEOREM 4.3. A nilsemigroup is subdirectly irreducible if and only if it contains a disjunctive zero and has the core.

Lemma 4.4. If a semigroup with a nontrivial annihilator contains a disjunctive element, then this semigroup has a disjunctive zero.

Proof. If 0 is disjunctive, there is nothing to prove. Let $k$ be a nonzero disjunctive element. $k$ is 0 -neat, so $G$ has the core. The core necessarily coincides with the annihilator, so $k$ is annihilating. Let $g_{1}$ and $g_{2}$ be two different elements. Then there exist $x$ and $y$ such that exactly one of elements $x g_{1} y, x g_{2} y$ is equal to $k$. Let $x g_{1} y=$ $k$. If $x g_{2} y \neq 0$, then for some $x_{1}, y_{1} x_{1} x g_{2} y y_{1}=k$, since $k=0$-neat. So $x_{1}$ and $y_{1}$ are not both void. The element $k$ is annihilating, therefore $x_{1} x g_{1} y y_{1}=x_{1} k y_{1}=0$. So 0 is disjunctive.

THEOREM 4.5. A nilsemigroup is subdirectly irreducible if and only if it contains a nonzero disjunctive element.

Proof. Such a semigroup has a core. The semigroup is subdirectly irreducible, by Lemmas 4.2, 4.4 and Theorem 4.3.

Homomorphic images of nilsemigroups are nilsemigroups, so every nilsemigroup is decomposable as a subdirect product of a family of subdirectly irreducible nilsemigroups.

A semigroup $G$ is called nilpotent if $G^{n}=0$ for some positive $n$. A subdirect product of nilpotent semigroups need not be nilpotent, but it is easy to prove that a semigroup $G$ is isomorphic to a subdirect product of a family of nilpotent semigroups if and only if the ideal $\cap G^{n}$ (for all positive $n$ ) is null.

THEOREM 4.6. A nilpotent semigroup is subdirectly irreducible if and only if it contains a disjunctive element.

Proof. By Theorem 4.5, it is sufficient to prove that a nilpotent semigroup with disjunctive zero is subdirectly irreducible.

If $G_{n}=0$ and $G^{n-1} \neq 0$, then $G^{n-1}$ is included in the annihilator 
of $G$. Let $k_{1}, k_{2}$ be nonzero annihilating elements. $x k_{1} y=0$ means. that $x$ or $y$ is not void. Therefore, $k_{1} \equiv k_{2}\left(\mathscr{C}_{0}\right)$, i.e., $k_{1}=k_{2}$. So $G^{n-1}$ is the annihilator containing exactly two elements. Clearly, $G^{n-1}$ is a core, hence $G$ is subdirectly irreducible, by Theorem 4.3.

COROLlaRY 4.6.1. A nilpotent semigroup is subdirectly irreducible if and only if it has a disjunctive zero.

THEOREM 4.7. Let $G$ be a subdirectly irreducible idempotent semigroup, $K$ be a core of $G$. If $G$ is a semigroup without zero, then one of the following two properties hold:

(1) $K$ is the set of all right zeros of $G$ and a left reductor.

(2) $K$ is the set of all left zeros of $G$ and a right reductor.

If $G$ has a zero, then the complementation of the zero is a subsemigroup satisfying (1) or (2).

Proof. Let $G$ have a zero. Then $K$ is 0 -simple, hence $K$ is a completely 0 -simple semigroup ([6], Corollary 2.56). Completely 0 -simple idempotent semigroups are rectangular bands with adjoined zeros (cf. [6], Exercise 2.7.9). Let $g_{1} g_{2}=0, g_{1} \neq 0, g_{2} \neq 0$. Then $K g_{1} K=K$ (see the last sentence of $\S 2$ ). Therefore $K g_{1} \neq 0$ and $g_{2} K \neq 0$, i.e., there exist $k_{1}, k_{2} \in K$ such that $k_{1} g_{1} \neq 0$ and $g_{2} k_{2} \neq 0$. So $k_{1} g_{1} \cdot g_{2} k_{2}=$ 0 . This equality contradicts to the fact that $K$ is a rectangular band with adjoined zero. Hence, $g_{1}=0$ or $g_{2}=0$, i.e., the complementation of 0 is a subsemigroup. This subsemigroup is subdirectly irreducible, by Theorem 3.6. $K \backslash\{0\}$ is the core of this subsemigroup, so it does not contain zero.

Now let $G$ be a subdirectly irreducible idempotent semigroup without zero. We have just seen that $K$ is a rectangular band. Define two equivalences $\varepsilon_{1}$ and $\varepsilon_{2}$ on $G . \quad g_{1} \equiv g_{2}\left(\varepsilon_{1}\right)$ means that $g_{1}=g_{2}$ or that $g_{1}, g_{2} \in K$ and $g_{1} g_{2}=g_{2}$ (the last equality implies $g_{2} g_{1}=g_{1}$ ). Let $g_{1} \equiv g_{2}\left(\varepsilon_{1}\right)$. If $g_{1}=g_{2}$, then $g g_{1} \equiv g g_{2}\left(\varepsilon_{1}\right)$ and $g_{1} g \equiv g_{2} g\left(\varepsilon_{1}\right)$. If $g_{1} \neq g_{2}$ then $g_{1}, g_{2} \in K$ and $g_{1} g_{2}=g_{2}$. Therefore $g g_{1} g g_{2}=g g_{1} g g_{1} g g_{2}=g g_{1} g_{2}=g g_{2}$ and $g g_{1} \equiv g g_{2}\left(\varepsilon_{1}\right) . \quad g_{1} g g_{1}=g_{1} \cdot g_{1} g g_{1} \cdot g_{1}=g_{1}$, since $K$ is a rectangular band and $g_{1} g g_{1} \in K$. Hence, $g_{1} g g_{2} g=g_{1} g g_{1} g_{2} g=g_{1} g_{2} g=g_{2} g$ and $g_{1} g \equiv$ $g_{2} g\left(\varepsilon_{1}\right)$. So $\varepsilon_{1}$ is a congruence. $\varepsilon_{2}$ is defined in a dual way $\left(g_{1} g_{2}=g_{1}\right.$, if $\left.g_{1}, g_{2} \in K\right)$. Clearly, $\varepsilon_{1} \cap \varepsilon_{2}=\Delta_{G}$. This means that $\varepsilon_{1}$ or $\varepsilon_{2}$ is identical, i.e., $K$ is a right zero semigroup or a left zero semigroup. Let $K$ be a right zero semigroup, i.e., $k_{1} k_{2}=k_{2}$ for $k_{1}, k_{2} \in K$. Then $g k=$ $g k \cdot k=k$ for every $k \in K$ and $g \in G$, i.e., $K$ is a set of right zeros of $G$. If $g$ is a right zero, then $g=K g \subset K$, i.e., $K$ is the set of all right zeros. Define $g_{1} \equiv g_{2}(\varepsilon)$ if and only if $k g_{1}=k g_{2}$ for all $k \in K$. $\varepsilon$ is a congruence, since $K$ is an ideal. Clearly, $\varepsilon \cap \varepsilon^{K}=\Delta_{G}$, so $\varepsilon=\Delta_{G}$. 
This means that $K$ is a left reductor.

Semigroups satisfying (2) were considered by E. S. Ljapin [11].

5. Commutative subdirectly irreducible semigroups. Commutative subdirectly irreducible rings have been described in [13]. We shall consider now commutative subdirectly irreducible semigroups. We distinguish three kinds of such semigroups.

Semigroups of the first kind are subdirectly irreducible abelian groups (with or without adjoined zero).

THEOREM 5.1. An abelian group is subdirectly irreducible if and only if it is a subgroup of $p^{\infty}$-group (i.e., if it is a $p^{\infty}$-group or a cyclic group of order $p^{n}$, where $p$ is a prime).

Proof. A subdirectly irreducible abelian group $G$ has a least nonunit subgroup $A$, by Theorem 3.2. Since $A$ does not contain any proper nonunit subgroup, it is a cyclic group of a prime order $p$. If $g \neq 1$ and $[g]$ is the cyclic subgroup of $G$ generated by $g$, then $A \subset[g]$, so $g$ is an element of a finite order $\mathrm{pm}$. Therefore the group $[g]$ has a subgroup of order $m$. In the same manner we prove that $m=1$ or $m$ is a multiple of $p$. So $g$ is an element of order $p^{n}$, i.e., $G$ is a $p$-group. It is well-known ([10], $\S 25$, p. 164) that every directly irreducible $p$-group is a $p^{\infty}$-group or cyclic. So $G$ coincides with one of the groups listed in Theorem 5.1. Clearly, these groups possess least nonunit subgroups and are subdirectly irreducible.

Thus, the concepts of direct and subdirect irreducibility are identical for periodic Abelian groups. However, these concepts differ in the general case. E.g., a directly irreducible Abelian torsion-free group is not subdirectly irreducible.

Semigroups of the first kind are exactly subdirectly irreducible commutative semigroups with globally idempotent cores. The complementation of zero in a commutative semigroup with globally idempotent core is a subsemigroup (otherwise the core is nilpotent). And a subdirectly irreducible commutative semigroup without zero and with globally idempotent core is a group, by Theorem 4.1 .

Semigroups of the second kind are subdirectly irreducible commutative semigroups having nontrivial annihilator. By Theorem 2.2, the core of such a semigroup is primitive and coincides with the annihilator. By Lemma 4.4,

THEOREM 5.2. A commutative semigroup with a nontrivial anni- 
hilator is subdirectly irreducible if and only if it contains a nonzero disjunctive element.

CoRollary 5.2.1. A commutative semigroup is a semigroup of the second kind if and only if contains a nonzero annihilating disjunctive element.

COROLlaRY 5.2.2. Subdirectly irreducible commutative nilsemigroups are semigroups of the second kind.

Subdirectly irreducible commutative semigroups different from the semigroups of the first two kinds are semigroups of the third kind. Hence, semigroups of the third kind are subdirectly irreducible commutative semigroups with a nilpotent core and trivial annihilator.

A divisor of zero is called nontrivial if it is different from zero. The set of all nondivisors of zero of a commutative semigroup is either empty or forms a subsemigroup.

THEOREM 5.3. A commutative semigroup is a semigroup of the third kind if and only if it contains an identity, a nontrivial divisor of zero and a nonzero disjunctive element, and the set of all nondivisors of zero forms a subdirectly irreducible group.

Proof. Let $G$ be a semigroup of the third kind with a core $K$, $F$ be the set of all elements annihilating the core, i.e., $f \in F \leftrightarrow K f=0$. Since $K^{2}=0, K \subset F$. Clearly, $F$ is an ideal. G is not of the second kind, so $K$ is not the annihilator. Therefore $G \neq F$. Let $A$ be the complementation of $F$ in $G, K_{0}$ be the set $K \backslash\{0\}, F_{0}$ be the set $F \backslash K$. So $\left\{\{0\}, K_{0}, F_{0}, A\right\}$ is a partition of $G$ ( $F_{0}$ may be empty). If $a \in A$, then $a K \neq 0$, i.e., $a K=K$, because $a K$ is an ideal of $G$. The set of all $g$ such that $a g=0$ forms an ideal. $K$ is not included in this ideal, therefore this ideal is null, i.e., $a$ is not a divisor of zero. Since elements of $F$ are divisors of zero, $A$ is the set of all nondivisors of zero. $G$ has the trivial annihilator, so for every $k \in K_{0} K=G K=$ $A k \cup\{0\}$, i.e., there exists such an element $e \in A$ that $e k=k$. By Theorem 3.5, $e$ is an identity of $G$. Let $a \in A$ and $k \in K_{0}$. Then $A a k=K_{0}$, so there exists $a_{1} \in A$ such that $a_{1} a k=k$. By Theorem 3.5, $a_{1} a=e$, so $a_{1}$ is the inverse of $a$ and $A$ is a subgroup of $G$. Let $a_{1} k=a_{2} k$ for $k \in K_{0}, a_{1}, a_{2} \in A$. Then $k=a_{1}^{-1} a_{2} k, a_{1}^{-1} a_{2}=e$ and $a_{1}=a_{2}$. $A k=K_{0}$, therefore the sets $A$ and $K_{0}$ have the same cardinality.

Let $\varepsilon$ be a congruence on a group $A$ generated by subsemigroup a. Define a binary relation $\varepsilon_{a}$ by the formula: $g_{1} \equiv g_{2}\left(\varepsilon_{a}\right) \leftrightarrow g_{1} \in g_{2} a$. It is easy to verify that $\varepsilon_{\mathfrak{a}}$ is a congruence on $G$ that induces on $A$ the congruence $\varepsilon$. Let $\left(\varepsilon_{i}\right)$ be a family of congruences on $A$ with 
identical intersection and $\left(\varepsilon_{\mathfrak{a}_{1}}\right)$ be the family of corresponding congruences on $G$. If $k_{1}, k_{2} \in K_{0}$, then $k_{1}=a k_{2}$ for some $a \in A$. Therefore $k_{1} \equiv k_{2}\left(\varepsilon_{\mathfrak{a}_{1}}\right)$ means that $k_{1} \in k_{2} \mathfrak{a}_{i}$ or that there exists $a_{i} \in \mathfrak{a}_{i}$ such that $k_{1}=k_{2} a_{i}$, or that $k_{2} a=k_{2} a_{i}$, or $a=a_{i}$, or $a \in a_{i}$. So $k_{i} \equiv k_{2}\left(\cap\left(\varepsilon_{a_{i}}\right)\right) \leftrightarrow$ $a \in \bigcap\left(\mathfrak{a}_{i}\right) \leftrightarrow a=1 \leftrightarrow k_{1}=k_{2}$. Therefore $\varepsilon^{K} \cap\left(\bigcap\left(\varepsilon_{\mathfrak{a}_{i}}\right)\right)=\Delta_{\theta}$ and, since $G$ is subdirectly irreducible, there exists $i$ such that $\varepsilon_{\mathfrak{a}_{i}}=\Delta_{G}$ and $\varepsilon_{i}=\Delta_{A}$. Hence, $A$ is subdirectly irreducible.

Now let $G$ be a commutative semigroup satisfying the conditions of our theorem. $G$ has a trivial annihilator and contains a nontrivial divisor of zero. Hence, $G$ is not a semigroup of the first or the second kind. It is sufficient to prove the subdirect irreducibility of $G$.

Let $A$ be the set of all nondivisors of zero, $k_{0}$ be a nonzero disjunctive element and $K$ the core of $G$ ( $K$ exists since $G$ has a nonzero disjunctive element). If $g$ is a nontrivial divisor of zero, then $g g_{1}=0$ for some $g_{1} \neq 0$. For every $k \in K$ there exist $x$ and $y$ such that $x g_{1} y=k$, so $g k=x g g_{1} y=0$ and $k$ is an annihilating element for the set $F=G \backslash A$, i.e., $F K=0$. If $A$ is one-element, then the set of all divisors of zero, i.e., the complementation of $A$, is a semigroup satisfying all conditions of Theorem 5.2, i.e., $G=H^{1}$ where $H$ is a semigroup of the second kind. $G$ is subdirectly irreducible, by Theorem 3.6.

Let $A$ have more than one element. Since $A$ is subdirectly irreducible, it has a least nonunit subsemigroup $a$. Let $\varepsilon$ be a nonidentical congruence on $G . \quad k_{0}$ does not form an $\varepsilon$-class, so there exists $g \neq k_{0}$ such that $k_{0} \equiv g(\varepsilon)$. If $g \notin K$, then for some $x, y x g y=k_{0}$, whence $x k_{0} y \equiv k_{0}(\varepsilon)$. $x$ and $y$ are not both void. If $x y \in A$, then $g=(x y)^{-1} k_{0} \in K$. So $x y \in A$ and $x k_{0} y=0$. Therefore $k_{0} \equiv 0(\varepsilon)$ and $a k_{0} \equiv 0(\varepsilon)$ for every $a \in \mathfrak{a}$, i.e.,

$$
\mathrm{a} k_{0} \times \mathrm{a} k_{0} \subset \varepsilon .
$$

If $g \in K_{0}$, then since $K=G k_{0}=A k_{0} \cup\{0\}, g=a_{0} k_{0}$ for some $a_{0} \in A$. In this case the set of all $a \in A$ such that $a k_{0} \equiv k_{0}(\varepsilon)$ forms a nonunit subgroup of $A$ (this subgroup contains $a_{0} \neq 1$ ). So $a$ is included in this subgroup and (5.1) is valid. Hence, (5.1) is always valid. Let $\varepsilon_{0}$ be the intersection of all nonidentical congruences on $G$. By formula (5.1), $a k_{0}$ is not divisible by $\varepsilon_{0}$, so $\varepsilon_{0}$ is not identical and $G$ is subdirectly irreducible, by Theorem 3.2 .

CoRollary 5.3.1. A semigroup $G$ is of the second kind if and only if $G^{1}$ is a semigroup of the third kind in which all elements different from identity are divisors of zero.

The "if" part has just been proved and the "only if" part follows from Theorems 3.6 and 5.2. 
COROLLARY 5.3.2. Every semigroup of the third kind has the following structure: it is the union of four mutually disjoint sets $\{0\}, K_{0}, F_{0}, A$, where $F_{0}$ may be empty, $K_{0}$ and $A$ are not empty. 0 is a zero, $A$ is the set of all nondivisors of zero; $A$ forms a subdirectly irreducible Abelian group; $K=K_{0} \cup\{0\}$ is the nilpotent core and $K F=0$, where $F=F_{0} \backslash K$. Sets $A$ and $K_{0}$ are of the same cardinality and $K_{0}$ is the set of all disjunctive elements of a semigroup (if $A$ is one-element, then 0 is also disjunctive). $A F_{0}=F_{0}$ and $F$ is an ideal of the semigroup. For every $a \in A$ and $k \in K_{0} a K_{0}=$ $K_{0}, A k=K_{0}$.

The greater portion of these propositions has been proved above. It follows from the formula $A_{k}=K_{0}$ that $K_{0}$ is the set of all disjunctive elements.

Note that semigroups of the third kind are a particular case of Rauter's "Übergruppen" [15].

A semigroup is called periodic if each of its elements generates a finite subsemigroup. Using the terminology of [4], we may say that semigroups of the third kind are extensions by semigroups of the first kind of semigroups all whose elements are divisors of zero. Periodic semigroups of the third kind are extensions by Abelian groups with zero of nilsemigroups. Finite commutative nilsemigroups are nilpotent, so subdirectly irreducible finite commutative semigroups are cyclic groups (possibly, with zero), finite nilpotent semigroups and extensions of nilpotent semigroups by cyclic groups with zero.

Though all subdirectly irreducible Abelian groups are periodic, this is not true for semigroups. We possess examples of nonperiodic semigroups of the second and of the third kinds.

6. Homomorphically simple semigroups. A semigroup is called homomorphically simple (or, $h$-simple) if it has only two congruences: identical and universal. Clearly, such semigroups are subdirectly irreducible. They have no proper nonnull ideals.

A subset $B$ of a set $A$ is proper, if $B \neq \varnothing$ and $B \neq A$.

THEOREM 6.1. A semigroup is h-simple if and only if each of its proper subsets is disjunctive.

Proof. Let $G$ be $h$-simple, $H$ be a proper subset of $G$. Clearly, $\mathscr{C}_{H}$ is not universal. So $\mathscr{C}_{H}=\Delta_{G}$, i.e., $H$ is disjunctive. If every proper subset of a semigroup is disjunctive, then the semigroup is $h$-simple, by Theorem 1.2.

THEOREM 6.2. A semigroup with zero is h-simple if and only 
if each of its elements is disjunctive.

Proof. Every element of an $h$-simple semigroup is disjunctive, by Theorem 6.1. If all elements of a semigroup with zero are disjunctive and $\varepsilon$ is a nonidentical congruence, then $\varepsilon\langle 0\rangle$ is a nonnull ideal. Every disjunctive element belongs to the core, so our semigroup has no proper nonnull ideals, i.e., $\varepsilon\langle 0\rangle$ coincides with the whole semigroup and $\varepsilon$ is universal.

It follows from Theorems 2.1 and 6.2:

CoRollary 6.2.1. A semigroup with zero is h-simple if and only if it is 0-simple and contains a disjunctive zero.

This condition is very similar to a somewhat more strong condition of L. M. Gluskin [8].

THEOREM 6.3. If a semigroup with disjunctive zero has a globally idempotent core, this core is an h-simple semigroup.

Proof. Let $k_{1}, k_{2}$ be distinct elements of a core $K$. Then there exist $x$ and $y$ such that exactly one of elements $x k_{1} y, x k_{2} y$ (say, $x k_{1} y$ ) is equal to $0 . K x k_{2} y K=K$ (cf. with the last sentence of $\S 2$ ). Therefore $k_{3} x k_{2} y k_{4} \neq 0$ and $k_{3} x k_{1} y k_{4}=0$ for some $k_{3}, k_{4} \in K$. Since $k_{3} x, y k_{4} \in K, 0$ is a disjunctive element of a semigroup $K . \quad K$ has no proper nonnull ideals (otherwise $K$ is nilpotent). Hence, $K$ is $h$-simple, by Corollary 6.2.1.

THEOREM 6.4. An h-simple noncommutative semigroup has no central elements different from zero and identity.

Proof. Let $e$ be a central element of a noncommutative $h$-simple semigroup $G$. Consider the congruence $\varepsilon_{(e)}$ constructed in the proof of Theorem 3.5. If $\varepsilon_{(e)}=\Delta_{A}$, then for every $g \in G g=g e$, since $g \equiv g e\left(\varepsilon_{\langle e\rangle}\right)$. Hence, $e$ is an identity of $G$.

Now let $\varepsilon_{(e)}$ be universal. Consider the congruence $\varepsilon_{e}: g_{1} \equiv g_{2}\left(\varepsilon_{e}\right) \leftrightarrow$ $g_{1} e=g_{2} e$. If $\varepsilon_{e}$ is universal, then $g_{1} e=g_{2} e$ for all $g_{1}, g_{2} \in G$. In particular, $g e=e^{2}$, i.e., the principal ideal generated by $e$ is $\left\{e, e^{2}\right\}$. $G$ is not commutative, so this ideal is null, i.e., $e$ is a zero of $G$. If $\varepsilon_{e}=$ $\Delta_{G}$, then $g_{1} e=g_{2} e \rightarrow g_{1}=g_{2}$. For every $g_{1}, g_{2}$ there exist $m$ and $n$ such that $g_{1} e^{m}=g_{2} e^{n}$. Let $m \geqq n$. Then $g_{1} e^{m-n}=g_{2}$ or $g_{1}=g_{2}$. In both cases $g_{1} g_{2}=g_{2} g_{1}$. But this is impossible ( $G$ is not commutative).

$H$-simple semigroups are examples of semigroups where every congruence is principal. Other examples of such semigroups are groups.

Let $H$ be a subset of a semigroup $G$ saturated for a congruence $\varepsilon$. 
Then $\varepsilon=C_{H}$ if and only if the factor-set $H / \varepsilon$ is a disjunctive set of a factor-semigroup $G / \varepsilon$. When $H$ is an $\varepsilon$-class, this was proved in $[21,23]$. We omit the analogous proof for the general case. As a consequence:

Proposition 6.5. A congruence $\varepsilon$ on a semigroup $G$ is principal if and only if the factor-semigroup $G / \varepsilon$ has a disjunctive subset.

It follows that the kernel of a homomorphism of $G$ on a subdirectly irreducible semigroup is a principal congruence generated by at least two different subsets of $G$ (these subsets are inverse images of disjunctive elements).

Another consequence is:

Proposition 6.6. Every congruence on a semigroup is principal if and only if every homomorphic image of this semigroup possesses a disjunctive subset.

7. Completely reductive and $f$-regular semigroups, A semigroup is called completely right (left) reductive if all its homomorphic images are right (left) reductive.

Clearly, every completely right reductive semigroup if right reductive.

Proposition 7.1. A commutative semigroup is not completely reductive if and only if it can be homomorphically mapped on a semigroup of the second kind.

Proof. Semigroups of the second kind are not reductive (they contain two distinct annihilating elements). On the other hand, if $G$ has no homomorphic images of the second kind, then every homomorphic image of $G$ is decomposable as a subdirect product of a family of semigroups of the first two kinds. Semigroups of these kinds have identities and are reductive. $G$ is completely reductive, by Theorem 3.8.

THEOREM 7.2. A commutative semigroup $G$ is completely reductive if and only if it satisfies one of the following equivalent conditions:

(1) $A G=A$ for every ideal $A$ of $G$.

(2) Every element of $G$ has a unit.

Proof. A semigroup of the second kind does not satisfy (1) if $A$ is the annihilator, and does not satisfy (2) because it has a nonzero annihilating element. If $G$ satisfies (1) or (2) then all homomorphic images of $G$ do. $G$ is completely reductive, by Proposition 7.1. 
Suppose $G$ does not satisfy (2). Let $g$ be an element of $G$ having no unit. Then $A G \neq A$ if $A=g G \cup g$. So $G$ does not satisfy (2). Consider the factor-semigroup $G / A G$. The subset $A / A G$ of this semigroup contains more than one element because $A G$ is a proper subset of $A . G / A G$ is not reductive because it contains different annihilating elements (all elements of $A / A G$ are annihilating). So $G$ is not completely reductive.

Corollary 7.2.1. A commutative periodic semigroup is completely reductive if and only if it satisfies one of the following conditions:

(1) The ideal generated by the set of all idempotents coincides with the semigroup.

(2) Every element has an idempotent unit.

Proof. Evidently $(2) \rightarrow(1)$. It is easy to prove $(1) \rightarrow(2)$, so both conditions are equivalent. (2) implies complete reductivity, by Theorem 7.2. If $G$ is completely reductive, periodic and commutative and $g \in G$, then $g h=g$ for some $h \in G$, by condition (2) of Theorem 7.2. So $h^{n}$ is a unit for $g$. But $h^{n}$ is idempotent for some $n$. So (2) holds.

CoRollary 7.2.2. A finite commutative semigroup is completely reductive if and only if it is globally idempotent, i.e., if and only if $G^{2}=G$.

Proof. If $G^{2} \neq G$, then $G$ does not satisfy condition (1) of Theorem 7.2 when $A=G$. If $G^{2}=G$ then every homomorphic image of $G$ is also globally idempotent. So $G$ cannot be mapped on a semigroup of the second kind, because finite semigroups of the second kind are nilpotent. $G$ is completely reductive, by Proposition 7.1.

THEOREM 7.3. The following properties of a semigroup $G$ are equivalent:

(1) The intersection of any two ideals of $G$ is equal to their product.

(2) Every ideal is globally idempotent.

(3) If $(g)$ is the principal ideal generated by an element $g \in \bar{G}$, then $g \in(g)^{2}$.

(4) Every subdirectly irreducible homomorphic image of $G$ has a globally idempotent core.

(5) Every homomorphic image of $G$ is decomposable as a subdirect product of a family of semigroups with globally idempotent cores.

Proof. $\quad(1) \rightarrow(2) \rightarrow(3) \rightarrow(4) \rightarrow(5) \rightarrow(1)$. We shall prove those 
implications that are not evident. (1) $\rightarrow(2)$ because $A \cap A=A$ for every ideal $A$. (3) is preserved under homomorphisms, so (3) $\rightarrow$ (4) because elements of a nilpotent core do not satisfy (3). Evidently, a subdirect product of semigroups with trivial annihilators has a trivial annihilator. A subdirectly irreducible semigroup with a globally idempotent core has a trivial annihilator. If $A$ and $B$ are ideals such that $A \cap B \neq A B$, then the factor-semigroup $G / A B$ has a nontrivial annihilator (elements of $A \supset B / A B$ are annihilating). So $(5) \rightarrow(1)$.

A semigroup satisfying conditions (1)-(5) of Theorem 7.3 is called $f$-regular. $f$-regular semigroups are an obvious analogue of $f$-regular rings that satisfy these conditions also [3].

Evidently, regular semigroups are $f$-regular. Homomorphic images of $f$-regular semigroups are $f$-regular.

\section{THEOREM 7.4 Commutative f-regular semigroups are regular.}

Proof. Let $A$ be a right ideal and $B$ a left ideal of a commutative $f$-regular semigroup $G$. Then $A$ and $B$ are ideals, so $A \cap B=A B$ Therefore $G$ is regular [9].

CoRollary 7.4.1. A semigroup is a commutative regular semigroup if and only if each of its homomorphic images is embeddable in a commutative regular semigroup.

\section{Proof. The "only if" part is evident.}

Clearly, subdirectly irreducible commutative regular semigroups are of the first kind (i.e., are periodic groups with or without zeros). Let $G$ be a subdirectly irreducible semigroup embeddable in a commutative regular semigroup $G_{1} . G_{1}$ is decomposable as a subdirect product of a family of periodic groups (with or without zeros). This decomposition induces a decomposition of $G$. But all decompositions of $G$ are trivial. So $G$ is embeddable in a periodic group (with or without zero). Hence, $G$ is a group (possibly, with a zero). Therefore $G$ has a globally idempotent core. Now let $G$ be a semigroup all of whose homomorphic images are embeddable in commutative regular semigroups. Since $G$ is a homomorphic image of itself, it is commutative. $G$ satisfies condition (4) of Theorem 7.3 , so it is $f$-regular. $G$ is a commutative regular semigroup, by Theorem 7.4 .

\section{REFERENCES}

1. G. Birkhoff, Subdirect unions in universal algebra, Bull. Amer. Math. Soc. 50 (1944), 764-768.

2. - Lattice theory, Amer. Math. Soc. Colloq. Publ. 25, Providence, 1948. 
3. R. L. Blair, Ideal lattices and the structure of rings, Trans. Amer. Math. Soc. 75 (1953), 136-153.

4. A. H. Clifford, Extensions of semigroups, Trans. Amer. Math. Soc. 68 (1950), 165173.

5. A. H. Clifford, and D. D. Miller, Semigroups having zeroid elements, Amer. J. Math. 70 (1948), 117-125.

6. A. H. Clifford, and G. B. Preston, The algebraic theory of semigroups, Amer. Math. Soc. Math. Surveys Vol. I, 7, Providence, 1961.

7. R. Croisot, Equivalences principales bilatères définies dans un demi-groupe, J. Math. pures et appl. (N.S.) 36 (1957), 373-417.

8. L. M. Gluskin, Simple semigroups with zero, Doklady AH SSSR 103 (1955), 5-8 (Russian).

9. K. Iséki, A characterisation of regular semi-group, Proc. Japan Acad. 32 (1956), 676-677.

10. A. G. Kuroš, Theory of groups, 2nd ed., Moscow, 1953 (Russian).

11. E. S. Ljapin, On certain representations of semigroups by transformations, "In the memory of N. G. Chebotarev" (a collection of papers), Kazan, 1964, pp. 60-66 (Russian).

12. E. S. Ljapin, Semigroups, Moscow, 1960 (Russian).

13. N. H. McCoy, Subdirectly irreducible commutative rings, Duke Math. J. 12 (1945), 381-387.

14. R. Pierce, Homomorphisms of semigroups, Ann. of Math. 59 (1954), 287-291.

15. H. Rauter, Abstrakte Kompositionssystem oder Übergruppen, J. reine u. angew. Math. 159 (1928), 229-237.

16. J. Riguet, Relations binaires, fermetures et correspondances de Galois, Bull. Soc. Math. France 78 (1948), 114-155.

17. B. M. Schein [Šăn], Embedding of semigroups in generalized groups, Matem. Sborn. (N. S.) 55 (1961), 379-400 (Russian).

18. - On subdirectly irreducible semigroups, Doklady AN SSSR 144 (1962), 999-1002; Correction, ibid. 148 (1963), 996 (Russian).

19. - On the theory of restrictive semigroups, Izv. vyš̌s. učebn. zaved., Matem. N2 (33) (1963), 152-154 (Russian).

20. M. P. Schützenberger. Une théorie algébrique du codage, C. R. Acad. Sci. Paris 242 (1956), 862-864.

21. M. Teissier, Sur les équivalences régulières dans les demi-groupes, C. R. Acad. Sci. Paris 232 (1951), 1987-1989.

22. G. Thierrin, Sur la structure des demi-groupes, Publs Sci. Univ. Alger. Sér. A3, 2 (1956), 161-171.

23. E. J. Tully, Jr., Representation of a semigroup by transformations of a set, Dissertation, Tulane University, 1960. 



\title{
PACIFIC JOURNAL OF MATHEMATICS
}

\author{
EDITORS
}

\section{H. SAMELSON}

Stanford University

Stanford, California

R. M. Blumenthal

University of Washington

Seattle, Washington 98105

\section{*J. DugundJI}

University of Southern California Los Angeles, California 90007

\section{RichaRd ARENS}

University of California

Los Angeles, California 90024

\section{ASSOCIATE EDITORS}
E. F. BECKENBACH
B. H. NEUManN
F. WOLF
K. YOSIDA

\section{SUPPORTING INSTITUTIONS}

\author{
UNIVERSITY OF BRITISH COLUMBIA \\ CALIFORNIA INSTITUTE OF TECHNOLOGY \\ UNIVERSITY OF CALIFORNIA \\ MONTANA STATE UNIVERSITY \\ UNIVERSITY OF NEVADA \\ NEW MEXICO STATE UNIVERSITY \\ OREGON STATE UNIVERSITY \\ UNIVERSITY OF OREGON \\ OSAKA UNIVERSITY \\ UNIVERSITY OF SOUTHERN CALIFORNIA
}

\author{
STANFORD UNIVERSITY \\ UNIVERSITY OF TOKYO \\ UNIVERSITY OF UTAH \\ WASHINGTON STATE UNIVERSITY \\ UNIVERSITY OF WASHINGTON \\ AMERICAN MATHEMATICAL SOCIETY \\ CHEVRON RESEARCH CORPORATION \\ TRW SYSTEMS \\ NAVAL ORDNANCE TEST STATION
}

\footnotetext{
Mathematical papers intended for publication in the Pacific Journal of Mathematics should be typewritten (double spaced). The first paragraph or two must be capable of being used separately as a synopsis of the entire paper. It should not contain references to the bibliography. Manuscripts may be sent to any one of the four editors. All other communications to the editors should be addressed to the managing editor, Richard Arens at the University of California, Los Angeles, California 90024 .

50 reprints per author of each article are furnished free of charge; additional copies may be obtained at cost in multiples of 50 .
}

The Pacific Journal of Mathematics is published monthly. Effective with Volume 16 the price per volume (3 numbers) is $\$ 8.00$; single issues, $\$ 3.00$. Special price for current issues to individual faculty members of supporting institutions and to individual members of the American Mathematical Society: $\$ 4.00$ per volume; single issues $\$ 1.50$. Back numbers are available.

Subscriptions, orders for back numbers, and changes of address should be sent to Pacific Journal of Mathematics, 103 Highland Boulevard, Berkeley 8, California.

Printed at Kokusai Bunken Insatsusha (International Academic Printing Co., Ltd.), No. 6, 2-chome, Fujimi-cho, Chiyoda-ku, Tokyo, Japan.

PUBLISHED BY PACIFIC JOURNAL OF MATHEMATICS, A NON-PROFIT CORPORATION

The Supporting Institutions listed above contribute to the cost of publication of this Journal, but they are not owners or publishers and have no responsibility for its content or policies.

* Paul A. White, Acting Editor until J. Dugundji returns. 


\section{Pacific Journal of Mathematics}

\section{Vol. 17, No. $3 \quad$ March, 1966}

Tsuyoshi Andô, Contractive projections in $L_{p}$ spaces ............... 391

Robert F. Brown, On a homotopy converse to the Lefschetz fixed point theorem ............................................... 407

Richard Albert Cleveland and Sandra Cleveland, On the multiplicative extension property................................ 413

Harold H. Johnson, An algebraic approach to exterior differential systems..................................... 423

Alan Cecil Lazer, The behavior of solutions of the differential equation

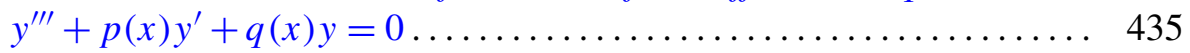

Judy Parr, Cohomology of cyclic groups of prime square order ......... 467

Donald Steven Passman, Groups whose irreducible representations have

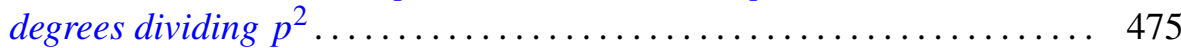

Ralph Tyrrell Rockafellar, Characterization of the subdifferentials of convex functions ........................................... 497

Donald Erik Sarason, Invariant subspaces and unstarred operator algebras...................................... 511

Donald Erik Sarason, Weak-star generators of $H^{\infty} \ldots \ldots \ldots \ldots \ldots \ldots . \ldots . \ldots$

Boris M. Schein, Homomorphisms and subdirect decompositions of semi-groups

Daniel Francis Shea, Jr., Functions analytic in a finite disk and having asymptotically prescribed characteristic

Zvi Ziegler, Generalized convexity cones 